Yetter-Drinfel'd modules for group-cograded multiplier Hopf algebras

Non Peer-reviewed author version

DELVAUX, Lydia (2008) Yetter-Drinfel'd modules for group-cograded multiplier Hopf algebras. In: COMMUNICATIONS IN ALGEBRA, 36(8). p. 2872-2882.

DOI: $10.1080 / 00927870802108080$

Handle: http://hdl.handle.net/1942/8473 


\title{
Yetter-Drinfel'd modules for group-cograded multiplier Hopf algebras
}

\author{
L. Delvaux ${ }^{1}$
}

\begin{abstract}
We give a representation-theoretic and a categorical interpretation of the Drinfel'd double into the framework of group-cograded multiplier Hopf algebras. The Drinfel'd double as constructed by Zunino for a finite-type Hopf group-coalgebra is an example of this construction in the sense that the components of the group-cograded multiplier Hopf algebras are unital and finite-dimensional algebras and the admissible action is related with the adjoint action of the group on itself.
\end{abstract}

Key words: Drinfel'd double, Hopf group-coalgebras, group-cograded multiplier Hopf algebras, Yetter-Drinfel'd modules.

Mathematics Subject Classification: 16W30, $17 \mathrm{~B} 37$.

\section{Introduction}

Let $G$ be any group. The prototype of a $G$-cograded multiplier Hopf algebra is given by the multiplier Hopf algebra $K(G)$ of complex valued functions with finite support in $G$. Recall that the product in $K(G)$ is pointwise. The algebra $K(G)$ has no unit, except where $G$ is finite. The multiplier algebra $M(K(G))$ of $K(G)$ is the largest algebra with unit in which $K(G)$ sits as a dense two-sided ideal. Clearly $M(K(G))$ is given by the algebra of all complex functions on $G$. The comultiplication $\Delta$ on $K(G)$ is given by the formula $(\Delta(f))(p, q)=f(p q)$ for all $f \in K(G)$ and $p, q \in G$. We have $\Delta(f) \in M(K(G) \otimes K(G))$. If $G$ is finite, the multiplier algebra $M(K(G) \otimes K(G))$ equals $K(G) \otimes K(G)$.

In this paper we work with more general $G$-cograded multiplier Hopf algebras in the sense of [A-De-VD, Definition 1.1]. Essentially, a multiplier Hopf algebra $B$ is $G$-cograded if there is a central, non-degenerate embedding $I: K(G) \rightarrow M(B)$. We require that $I$ respects the comultiplication in the sense that $\Delta(I(f))=(I \otimes I)(\Delta(f))$

\footnotetext{
${ }^{1}$ Department of Mathematics, Universiteit Hasselt, Agoralaan, B-3590 Diepenbeek, Belgium. E-mail: lydia.delvaux@uhasselt.be
} 
for all $f \in K(G)$. On the left hand side, we have extended the homomorphism $\Delta$ from $B$ to the multiplier algebra $M(B)$, in the sense of [VD1-A5]. Similary, on the right hand side, we have extended the homomorphism $I \otimes I$ from $K(G) \otimes K(G)$ to $M(K(G) \otimes K(G))$. A $G$-cograded multiplier Hopf algebra is denoted as $B=\bigoplus_{p \in G} B_{p}$ where $B_{p}$ are algebras with a non-degenerate product. For all $p, q \in G$ we have $\Delta\left(B_{p q}\right)\left(1 \otimes B_{q}\right)=B_{p} \otimes B_{q}$. Observe that the multiplier algebra $M(B)=\prod_{p \in G} M\left(B_{p}\right)$. It is shown in [A-De-VD] that a Hopf group-coalgebra as introduced by Turaev in $[\mathrm{T}]$, is a special case of a group-cograded multiplier Hopf algebra. Therefore, a lot of results for Hopf group-coalgebras follow from the more general results of multiplier Hopf algebras. E.g. the Drinfel'd double as constructed in [Z1] is an example of the Drinfel'd double construction $D^{\pi}$ in [De-VD3, Theorem 3.8]. In the paper [De-VD3], we consider any group-cograded multiplier Hopf algebra $B$ with an admissible action of the group. If we take the components of $B$ as unital finite-dimensional algebras and we require the admissible action to be a "crossing", we recover the construction as given in [Z1].

For convenience of the reader, we recall the construction of the Drinfel'd double $D^{\pi}$. We start with a $G$-cograded multiplier Hopf algebra $B$. So $B$ has the form $B=\bigoplus_{p \in G} B_{p}$. Assume that there is a group homomorphism $\pi: G \rightarrow \operatorname{Aut}(B)$, where Aut $(B)$ denotes the group of algebra automorphisms on $B$.

We call $\pi$ an admissible action of $G$ on $B$ if also the following requirements hold

(1) $\Delta\left(\pi_{p}(b)\right)=\left(\pi_{p} \otimes \pi_{p}\right)(\Delta(b))$ for all $b \in B$

(2) $\pi_{p}\left(B_{q}\right)=B_{\rho_{p}(q)}$ where $\rho$ is an action of the group $G$ on itself

(3) $\pi_{\rho_{p}(q)}=\pi_{p q p^{-1}}$

This means that the map $\pi$ takes care of $\rho$ not being the adjoint action. If $\rho$ is the adjoint action itself, $\pi$ is called a crossing.

Take $B$ and $\pi$ as above. We consider a new regular multiplier Hopf algebra on $B$ by deforming the comultiplication while the algebra structure on $B$ is kept. The deformation of the comultiplication of $B$ depends on the action $\pi$, in the following way

$$
\begin{aligned}
& \widetilde{\Delta}(b)\left(1 \otimes b^{\prime}\right)=\left(\pi_{q^{-1}} \otimes \iota\right)\left(\Delta(b)\left(1 \otimes b^{\prime}\right)\right) \\
& \left(1 \otimes b^{\prime}\right) \widetilde{\Delta}(b)=\left(\pi_{q^{-1}} \otimes \iota\right)\left(\left(1 \otimes b^{\prime}\right) \Delta(b)\right)
\end{aligned}
$$

for all $b \in B$ and $b^{\prime} \in B_{q}$. 
Further we assume that $\langle A, B\rangle$ is a pairing of two regular multiplier Hopf algebras, in the sense of [Dr-VD]. As before, $B$ is $G$-cograded and $\pi$ is an admissible action of $G$ on $B$. We consider a twisted tensor product algebra on the tensor product $A \otimes B$. This means that the trivial flip map is replaced by a more general twist map $R: B \otimes A \rightarrow A \otimes B$. This map $R$ satisfies the appropriate compatibility conditions with respect to the multiplications on $A$ and $B$. The twist map $R$ depends on the pairing $\langle A, B\rangle$, as well as on the action $\pi$. For an explicit expression of the formula $R(b \otimes a)$, we refer to [De-VD3, Definition 3.4]. The algebra defined in this way is denoted as $A \bowtie B$. Finally, this algebra has the structure of a regular multiplier Hopf algebra if we consider the comultiplication $\bar{\Delta}$ on $A \bowtie B$ where $\bar{\Delta}(a \bowtie b)=\Delta^{c o p}(a) \widetilde{\Delta}(b)$ in $M((A \bowtie B) \otimes(A \bowtie B))$. We observe that for $a \in A$, we use the opposite comultiplication of $A$. For $b \in B$, we use the deformation $\widetilde{\Delta}(b)$ as defined above.

In this paper, we characterize the modules of $D^{\pi}$. This is done from the point of view that $D^{\pi}$ is a non-trivial twisted tensor product on the space $A \otimes B$, see above. We require a natural condition on the pairing $\langle A, B\rangle$ which generalizes the dual bases for finite-dimensional Hopf algebras. Then the characterization of the left modules over $D^{\pi}$ can be rephrased purely in terms of the multiplier Hopf algebra $B$, without any reference to the multiplier Hopf algebra $A$. The compatibility conditions for these $\pi$-Yetter-Drinfel'd modules over $B$ are given in Theorem 2.1. When we require the admissible action to be a crossing (this means that $\pi$ is related with the adjoint action of the group $G$ on itself) and we assume that the components are unital and finite-dimensional, the characterization in Theorem 2.1 can be put in the setting of [Z2, Section 8]. We notice that in this special situation our Drinfel'd double construction is isomorphic with the so-called mirror construction, given in [Z2, Section 9]. If $\pi$ is a crossing of the group $G$ on an arbitrary $G$-cograded multiplier Hopf algebra $B$, we have that the Drinfel'd double $D^{\pi}$ is again $G$-cograded and there is a natural crossing of $G$ on $D^{\pi}$, see [De-VD3, Proposition 3.13]. Furthermore, we have that $D^{\pi}$ is $\pi$-quasitriangular, see [De-VD-W, Theorem 3.12]. The categorical interpretation of this quasitriangularity is translated to the $\pi$-Yetter-Drinfel'd modules over $B$, see Theorem 3.1. Our braiding is in the sense of the centre-construction of a category as given in [K, Sections XIII 4-5].

All algebras are considered over the field $\mathbb{C}$. We do not assume that an algebra $A$ has a unit. But we require that the multiplication, considered as a bilinear map is non-degenerate. The multiplier algebra, denoted as $M(A)$, is the largest algebra 
with a unit in which $A$ is contained as a dense two-sided ideal. The identity in any (multiplier) algebra is denoted by 1 . The identity map is denoted as $\iota$.

For a regular multiplier Hopf algebra $A$ (i.e. with a bijective antipode) we denote the comultiplication by $\Delta$. Observe that $\Delta: A \rightarrow M(A \otimes A)$. However, by the defining conditions on $\Delta$, we have for all $a, b \in A$ that $\Delta(a)(1 \otimes b), \Delta(a)(b \otimes 1)$, $(1 \otimes b) \Delta(a)$ and $(b \otimes 1) \Delta(a)$ are elements in $A \otimes A$. It can be motivated, see e.g. [Dr-VD-Z, Section 2] that these elements are denoted by Sweedler notation, e.g. $\Delta(a)(1 \otimes b)=\sum a_{(1)} \otimes a_{(2)} b$. In an expression, denoted by Sweedler notation, one always has to make sure that at most one factor $a_{(k)}$ is not multiplied ("covered") by an element in $A$.

When we consider a module $V$ over an algebra $A$, we always mean a left module which is unital. A (left) $A$-module $V$ is unital if $A \triangleright V=V$. By the regularity conditions on $A$, this implies that for all $x \in V$, we have an element $e \in A$ such that $x=e \triangleright x$. For details, we refer to [Dr-VD-Z, Section 3]. Therefore, the comultiplication on $A$ can be used to make the category of (left) $A$-modules into a tensor category with unit.

\section{Basic references}

The material needed for reading this paper is given in the following basic references. For (regular) multiplier Hopf algebras, we refer to [VD1] and [VD-Z1]. The group-cograded multiplier Hopf algebras are introduced in [A-De-VD] and studied in [De-VD-W]. They generalize the Hopf group-coalgebras, as introduced by Turaev in $[T]$. The Drinfel'd double construction into the framework of multiplier Hopf algebras is associated to a pairing, see [Dr-VD] and [De-VD1]. To have the analogous properties as for the Drinfel'd double of a finite-dimensional Hopf algebra, we assume that the pairing $\langle A, B\rangle$ of two multiplier Hopf algebras has a canonical multiplier $W \in M(A \otimes B)$. Essentially, the multiplier $W$ takes the role of the dual bases in the finite-dimensional case. For details, we refer to [De-VD2, Section 4]. The Drinfel'd double construction for group-cograded multiplier Hopf algebras is done in [De-VD3]. 


\section{$2 \pi$-Yetter-Drinfel'd modules}

Let $\langle A, B\rangle$ be a pair of multiplier Hopf algebras. Let $G$ denote a group and assume that $B$ is $G$-cograded. As an algebra, we write $B=\bigoplus_{p \in G} B_{p}$. Let $\pi$ be an admissible action of $G$ on $B$, in the sense of [De-VD3, Definition 2.6]. So for all $p \in G$, we have an automorphism $\pi_{p}$ on $B$ which respects the multiplication and the comultiplication of $B$. Furthermore for all $p, q \in G$, we have $\pi_{p}\left(B_{q}\right)=B_{\rho_{p}(q)}$ where $\rho$ is an automorphism of $G$ on itself. We require that $\pi_{\rho_{p}(q)}=\pi_{p q p^{-1}}$ for all $p, q \in G$. In the framework of Hopf group-coalgebras, one sets $\rho_{p}(q)=p q p^{-1}$ for all $p, q \in G$, see [T]. Let $D^{\pi}$ denote the Drinfel'd double as constructed in [De-VD3, Theorem 3.8]. As an algebra, $D^{\pi}$ is a twisted tensor product on the linear space $A \otimes B$. Therefore, a left $D^{\pi}$-module is nothing but a linear space $V$ with a left $B$-module structure, denoted as $B . V$, as well as a left $A$-module structure, denoted as $A \triangleright V$. For all $a \in A, b \in B_{p}$ and $x \in V$, the following compatibility equation yields

$$
b \cdot(a \triangleright x)=\sum a_{i} \triangleright\left(b_{i} \cdot x\right)
$$

where $\sum a_{i} \otimes b_{i}=T(b \otimes a)=\sum\left(\pi_{p^{-1}}\left(b_{(1)}\right) \triangleright a \triangleleft S^{-1}\left(b_{(3)}\right)\right) \otimes b_{(2)}$.

The actions $\downarrow$ and $\varangle$ are the regular actions of $B$ on $A$, associated to the pairing $\langle A, B\rangle$, see [Dr-VD].

We rephrase the above compatibility condition in terms of the multiplier Hopf algebra $B$, without any reference to the paired multiplier Hopf algebra $A$. We need the notion of a right- $B$-comodule. In Hopf algebra theory, it is possible to define the structure of a comodule on a vector space. In the setting of multiplier Hopf algebras however, more structure is needed. In [VD-Z2], the setting is that of an algebra $V$ and a regular multiplier Hopf algebra $B$. Then, a right coaction of $B$ on $V$ is an injective linear map $\Gamma: V \rightarrow M(V \otimes B)$ satisfying

(i) $\Gamma(V)(1 \otimes B) \subseteq V \otimes B$ and $(1 \otimes B) \Gamma(V) \subseteq V \otimes B$

(ii) $(\Gamma \otimes \iota) \Gamma=(\iota \otimes \Delta) \Gamma$

The algebra structure of $V$ is needed to be able to consider the multiplier algebra $M(V \otimes B)$. It would be too restrictive to assume that the coaction $\Gamma$ has range in the tensor product itself.

Observe that Condition (i) is used to give a meaning to the left hand side of the equation in Condition (ii). The link between left $A$-modules and right $B$-comodules is given by the so-called canonical multiplier $W$ in $M(A \otimes B)$, in the sense of [De-VD2, 
Section 4] and [De2, Section 2]. A multiplier $W$ in $M(A \otimes B)$ is called canonical for the pairing $\langle A, B\rangle$ if $W$ is invertible in $M(A \otimes B)$ and if $\langle W, a \otimes b\rangle=\langle a, b\rangle$ for all $a \in A$ and $b \in B$. Let $B$ be a finite-dimensional Hopf algebra and consider $A=B^{\prime}$ where $B^{\prime}$ denotes the dual Hopf algebra of $B$. If $\left\{f_{i}\right\} \subset B^{\prime}$ and $\left\{e_{i}\right\} \subset B$ are dual bases, then $W=\sum f_{i} \otimes e_{i}$ is the canonical element in $B^{\prime} \otimes B$ for the natural pairing $\left\langle B^{\prime}, B\right\rangle$.

2.1 Theorem Consider the notations and the assumptions as above. We have that $V$ is a (left) $D^{\pi}$-module if and only if $V$ is a left $B$-module for the action $B . V$ and $V$ is a right $B$-comodule for the right coaction $\Gamma: V \rightarrow M(V \otimes B)$ such that the left action and the right coaction of $B$ on $V$ satisfy the compatibility relation

$$
\sum\left(d_{(1)} \cdot \otimes c d_{(2)}\right) \Gamma(v)=\sum(1 \otimes c) \Gamma\left(d_{(2)} \cdot v\right)\left(1 \otimes \pi_{p^{-1}}\left(d_{(1)}\right)\right)
$$

for all $v \in V, c \in B_{q}$ and $d \in B_{p q}$ (where $\left.p, q \in G\right)$.

Proof. In [De2, Theorem 2.3], we have proven that a left $A$-module $V$ is determined by a unique right $B$-comodule structure on $V$ in the following way. Let $A \triangleright V$ denote a left $A$-module, then there is a right $B$-comodule $\Gamma: V \rightarrow M(V \otimes B)$ so that

$$
a \triangleright v=(\iota \otimes\langle a, \cdot\rangle) \Gamma(v)
$$

for all $A \in A$ and $v \in V$.

On the right hand side of the above equation, we have that $\Gamma(v)$ sits in the multiplier algebra $M(V \otimes B)$. However, by the regularity conditions on the pairing $\langle A, B\rangle$, there is an element $b \in B$ such that the right hand side should be read as $(\iota \otimes\langle a, \cdot\rangle)(\Gamma(v)(1 \otimes b))$. As we assume for the coaction $\Gamma$ that $\Gamma(V)(1 \otimes B) \subseteq V \otimes B$, the expression $(\iota \otimes\langle a, \cdot\rangle)(\Gamma(v)(1 \otimes b))$ determines an element in $V$. The compatibility condition between the left $B$-module structure and the left $A$-module structure on a left $D^{\pi}$-module $V$ can be rephrased as follows. For all $a \in A, b \in B_{p}$ and $v \in V$, we have

$$
\begin{aligned}
& \left(\iota_{V} \otimes\langle a, \cdot\rangle\right)(b \cdot \otimes 1) \Gamma(v)=b \cdot\left(\left(\iota_{V} \otimes\langle a, \cdot\rangle\right) \Gamma(v)\right)=b \cdot(a \triangleright v)=\sum a_{i} \triangleright\left(b_{i} \cdot v\right) \\
& =\sum\left(\iota_{V} \otimes\left\langle a_{i}, \cdot\right\rangle\right) \Gamma\left(b_{i} \cdot v\right)=\sum\left(\iota_{V} \otimes\left\langle\pi_{p^{-1}}\left(b_{(1)}\right) \triangleright a \triangleleft S^{-1}\left(b_{(3)}\right), \cdot\right\rangle\right) \Gamma\left(b_{(2)} \cdot v\right) \\
& =\sum\left\langle a_{(1)}, S^{-1}\left(b_{(3)}\right)\right\rangle\left\langle a_{(3)}, \pi_{p^{-1}}\left(b_{(1)}\right)\right\rangle\left(\iota_{V} \otimes\left\langle a_{(2)}, \cdot\right\rangle\right) \Gamma\left(b_{(2)} \cdot v\right) \\
& =\left(\iota_{V} \otimes\langle a, \cdot\rangle\right)\left(\sum\left(1 \otimes S^{-1}\left(b_{(3)}\right)\right) \Gamma\left(b_{(2)} \cdot v\right)\left(1 \otimes \pi_{p^{-1}}\left(b_{(1)}\right)\right)\right) .
\end{aligned}
$$


Observe that the above equations are in $V \otimes B$. All decompositions are well-covered by the use of the regularity conditions on the pairing $\langle A, B\rangle$. As the pairing is a non-degenerate linear form on $A \otimes B$, we obtain the following equation in $V \otimes B$. For any $p, q \in G$ and $b \in B_{p}, b^{\prime} \in B_{q}$, we have for all $v \in V$

$$
\left(b \cdot \otimes b^{\prime}\right) \Gamma(v)=\sum\left(1 \otimes b^{\prime} S^{-1}\left(b_{(3)}\right)\right) \Gamma\left(b_{(2)} \cdot v\right)\left(1 \otimes \pi_{p^{-1}}\left(b_{(1)}\right)\right) .
$$

From the axioms on a regular multiplier Hopf algebra, we have $(1 \otimes B) \Delta(B)=B \otimes B$, see [VD1]. By the use of [VD1, Lemma 5.5], the equation above is equivalent to the following statement. For any $p, q \in G$ and $d \in B_{p q}, c \in B_{q}$ we have for all $v \in V$

$$
\left(\sum d_{(1)} \cdot \otimes c d_{(2)}\right) \Gamma(v)=\sum(1 \otimes c) \Gamma\left(d_{(2)} \cdot v\right)\left(1 \otimes \pi_{p^{-1}}\left(d_{(1)}\right)\right) .
$$

By the use of the $G$-cograding and the admissible action $\pi$, we have that in the left hand side $(1 \otimes c) \Delta(d) \in B_{p} \otimes B_{q}$. In the right hand side we have $\left(\pi_{p}(c) \otimes 1\right) \Delta(d) \in$ $B_{\rho_{p}(q)} \otimes B_{\rho_{p}\left(q^{-1}\right) p q}$

2.2 Definition Let $B$ be a $G$-cograded multiplier Hopf algebra and let $\pi$ be an admissible action of $G$ on $B$. An algebra $V$ with a left $B$-module structure and a right $B$-comodule structure is called a $\pi$-Yetter-Drinfel'd module if the compatibility condition of Theorem 2.1 is satisfied. The set of all $\pi$-Yetter-Drinfel'd modules over $B$ is denoted as ${ }_{B} \pi(\mathcal{Y D})^{B}$.

In the characterization of Theorem 2.1, we have dispensed with the Drinfel'd double $D^{\pi}$. So we do not need to assume that $B$ is paired with another multiplier Hopf algebra to define the $\pi$-Yetter-Drinfel'd modules over $B$.

2.3 Remark Let $B$ be a finite-type Hopf group-coalgebra and assume that $\pi$ is a crossing, i.e. $\pi_{p}\left(B_{q}\right)=B_{p q p^{-1}}$ for all $p, q \in G$. In Theorem 2.1, the multiplier Hopf algebra $A$ can be taken as the (usual) Hopf algebra $B^{*}=\bigoplus_{p \in G}\left(B_{p}\right)^{\prime}$, where $\left(B_{p}\right)^{\prime}$ denotes the linear dual of $B_{p}$. The formula in Theorem 2.1 is now given as in [Z2, Section 8]. When $G$ is given by the trivial group, we recover the well-known characterization of Yetter-Drinfel'd modules for a finite-dimensional Hopf algebra. In these settings, we don't need an underlying algebra structure on the YetterDrinfel'd modules because the comultiplication of $B$ is a map $\Delta: B \rightarrow B \otimes B$. Furthermore, there is always a canonical multiplier $W$ in $M\left(B^{*} \otimes B\right)$. More precisely, $W=\sum_{p \in G} f_{p, i} \otimes e_{p, i}$ where for all $p \in G$, the sets $\left\{f_{p, i}\right\}$ in $\left(B_{p}\right)^{\prime}$ and $\left\{e_{p, i}\right\}$ in $B_{p}$ are dual bases. 


\section{The braided monoidal category ${ }_{B} \pi(\mathcal{Y} \mathcal{D})^{B}$}

As before, we consider a multiplier Hopf algebra $B$ which is cograded by a group $G$. As an algebra we have $B=\bigoplus_{p \in G} B_{p}$ where $B_{p}$ is a subalgebra with a non-degenerate product. Let $\pi$ denote an admissible action of $G$ on $B$. We consider the category ${ }_{B} \pi(\mathcal{Y D})^{B}$ of $\pi$-Yetter-Drinfel'd modules over $B$, in the sense of Definition 2.2. The morphisms in ${ }_{B} \pi(\mathcal{Y D})^{B}$ are linear maps which are left $B$-module morphisms as well as right $B$-comodule morphisms.

If $B$ is paired with a multiplier Hopf algebra $A$, we have proven in Theorem 2.1 that the category ${ }_{B} \pi(\mathcal{Y} \mathcal{D})^{B}$ is given by the left unital modules over the Drinfel'd double $D^{\pi}$, associated to the pair $\langle A, B\rangle$. We made use of the canonical multiplier $W$ in $M(A \otimes B)$. The morphisms between left $D^{\pi}$-modules, correspond to the morphisms in ${ }_{B} \pi(\mathcal{Y D})^{B}$, use [De2, Theorem 2-3]. By the bialgebra structure on $D^{\pi}$, the modules over $D^{\pi}$ have the structure of a monoidal tensor category. Therefore, it is expected that the category ${ }_{B} \pi(\mathcal{Y} \mathcal{D})^{B}$ is also a monoidal tensor category. Let $V$ be in ${ }_{B_{p}} \pi(\mathcal{Y} \mathcal{D})^{B}$ and let $V^{\prime}$ be in ${ }_{B_{q}} \pi(\mathcal{Y D})^{B}$, then $V \otimes V^{\prime}$ is in ${ }_{B_{p_{q}(p) q}} \pi(\mathcal{Y D})^{B}$ in the following way

$$
b \cdot\left(v \otimes v^{\prime}\right)=\sum \pi_{q^{-1}}\left(b_{(1)}\right) \cdot v \otimes b_{(2)} \cdot v^{\prime}
$$

for all $b \in B_{\rho_{q}(p) q}, v \in V$ and $v^{\prime} \in V^{\prime}$. To determine the right $B$-comodule structure on the tensor product $V \otimes V^{\prime}$, we translate the $A$-module structure on $V \otimes V^{\prime}\left(A^{\text {cop }}\right.$ is embedded in $\left.D^{\pi}\right)$. This translation is done by the use of the canonical multiplier in $M(A \otimes B)$. We have denoted this multiplier by the letters $W$ and $P$. For $a \in A$ and $b \in B$, we write $(a \otimes 1) W(1 \otimes b)$ as $\sum a W^{(1)} \otimes W^{(2)} b$ in $A \otimes B$. Following [De2, Proposition 2.2], we have for all $b \in B, v \in V$ and $v^{\prime} \in V^{\prime}$

$$
\begin{aligned}
& \Gamma\left(v \otimes v^{\prime}\right)(1 \otimes 1 \otimes b)=\sum\left(W^{(1)} \triangleright\left(v \otimes v^{\prime}\right)\right) \otimes W^{(2)} b \\
& =\sum\left(P^{(1)} \triangleright v\right) \otimes\left(W^{(1)} \triangleright v^{\prime}\right) \otimes W^{(2)} P^{(2)} b .
\end{aligned}
$$

We made use of the formula $(\Delta \otimes \iota)(W)=W^{13} W^{23}$. We have obtained the following right $B$-comodule structure on $V \otimes V^{\prime}$

$$
\Gamma\left(v \otimes v^{\prime}\right)(1 \otimes 1 \otimes b)=\Gamma\left(v^{\prime}\right)_{23} \Gamma(v)_{13}(1 \otimes 1 \otimes b)
$$

for all $v \in V, v^{\prime} \in V^{\prime}$ and $b \in B$. In the right hand side, we use the leg-numbering notation in the usual way. 
One can check that the compatibility condition holds for the tensor object $V \otimes V^{\prime}$. Moreover, we have that ${ }_{B} \pi(\mathcal{Y D})^{B}$ is a monoidal category. We omit these proofs because we would be repeating the construction of $D^{\pi}$ as bialgebra, see [De-VD3].

So far, we have "translated" the multiplier Hopf algebra structure on $D^{\pi}$ to determine the monoidal category ${ }_{B} \pi(\mathcal{Y} \mathcal{D})^{B}$. Further structures on the multiplier Hopf algebra $D^{\pi}$ will correspond directly to properties of its category of modules and can be translated towards the category ${ }_{B} \pi(\mathcal{Y D})^{B}$. Further in this sequel, we assume that the admissible action of $G$ on $B$ is given as a crossing. This means that $\pi_{p}\left(B_{q}\right)=B_{p q p^{-1}}$ for all $p, q \in G$. However, the components of the $G$-cograded multiplier Hopf algebra $B$, denoted as $B_{p}$ for all $p \in G$, are arbitrary algebras with a non-degenerate multiplication. In this setting, we have that $D^{\pi}$ is $G$-cograded and there is a natural crossing of $G$ on $D^{\pi}$. More precisely, in [De-VD3, Proposition 3.13], we have proven that $D^{\pi}$ is $G$-cograded as follows

$$
D^{\pi}=\bigoplus_{p \in G}\left(D^{\pi}\right)_{p} \quad \text { with }\left(D^{\pi}\right)_{p}=A \bowtie B_{p^{-1}} .
$$

For all $p \in G$, define $\pi_{p}^{\prime}$ on $A$ via the formula $\left\langle\pi_{p}^{\prime}(a), b\right\rangle=\left\langle a, \pi_{p^{-1}}(b)\right\rangle$ for all $a \in A$, $b \in B$. Then, the maps $\pi_{p}^{\prime} \otimes \pi_{p}$ define a crossing of $G$ on $D^{\pi}$. Let $W$ in $M(A \otimes B)$ denote the canonical multiplier of the pair $\langle A, B\rangle$. Let $\sigma$ be the twist map on $A \otimes B$, extended to $M(A \otimes B)$. It is proven in [De-VD-W, Theorem 3.12] that the embedding $\sigma(W)$ in $M\left(D^{\pi} \otimes D^{\pi}\right)$ is a generalised $\pi$-matrix for $D^{\pi}$, in the sense of [De-VD-W, Definition 3.1]. By the use of [De-VD-W, Section 3.4], this $\pi$-quasitriangularity of $D^{\pi}$ corresponds to the following properties of the category of (left) modules over $D^{\pi}$. For all $p \in G$, let ${ }_{p} \mathcal{M}$ denote the modules over the algebra $\left(D^{\pi}\right)_{p}$. Then we have that the category of left modules over $D^{\pi}$ is given as ${ }_{D^{\pi}} \mathcal{M}=\prod_{p \in G}{ }_{p} \mathcal{M}$. For all $p \in G$, there is an invertible functor $F_{p}$ on ${ }_{D^{\pi}} \mathcal{M}$. If $V \in{ }_{q} \mathcal{M}$, then $F_{p}(V) \in{ }_{p q p^{-1}} \mathcal{M}$. As a linear space, we have that $F_{p}(V)$ equals $V$. Let the $D^{\pi}$-module structure on $V$ be denoted as $D^{\pi} \rightarrow V$. For an element $(a \bowtie b) \in\left(D^{\pi}\right)_{p q p^{-1}}$, we have $(a \bowtie b) \rightarrow F_{p}(v)=F_{p}\left(\left(\pi_{p^{-1}}^{\prime}(a) \bowtie \pi_{p^{-1}}(b)\right) \rightarrow v\right)$. A morphism in ${ }_{q} \mathcal{M}$ is sent to itself, now considered as a morphism in ${ }_{p q p^{-1}} \mathcal{M}$.

Finally, the $\pi$-quasitriangularity of $D^{\pi}$ gives the following $\pi$-braiding in ${ }_{D \pi} \mathcal{M}$. Let $V\left(\right.$ resp. $\left.V^{\prime}\right)$ be in ${ }_{p} \mathcal{M}$ (resp. $\left.{ }_{q} \mathcal{M}\right)$. Then we have

$$
\begin{aligned}
& t_{V, V^{\prime}}: V \otimes V^{\prime} \rightarrow F_{p}\left(V^{\prime}\right) \otimes V \text { such that } \\
& t_{V, V^{\prime}}\left(v \otimes v^{\prime}\right)=\sum F_{p}\left(W^{(1)} \triangleright v^{\prime}\right) \otimes\left(W^{(2)} \cdot v\right)
\end{aligned}
$$

where $A \triangleright V$ (resp. $B \cdot V$ ) denotes the $A$-module (resp. $B$-module) structure on $V$. 
In Theorem 2.1, we have given a characterization for the $\pi$-Yetter-Drinfel'd modules, without the use of a pairing and a Drinfel'd double. So, associated to any $G$-cograded multiplier Hopf algebra $B$ and a crossing $\pi$ of $G$ on $B$, we have the following $\pi$-braided monoidal tensor category ${ }_{B} \pi(\mathcal{Y} \mathcal{D})^{B}$.

3.1 Theorem Let $B$ be a $G$-cograded multiplier Hopf algebra and let $\pi$ denote a crossing of $G$ on $B$. The monoidal category ${ }_{B} \pi(\mathcal{Y} \mathcal{D})^{B}$ is $\pi$-braided.

Proof. Let $V$ be in ${ }_{B} \pi(\mathcal{Y D})^{B}$. The left action of $B$ on $V$ is denoted as $B \cdot V$. The right coaction of $B$ on $V$ is denoted as $\Gamma: V \rightarrow M(V \otimes B)$. If $B$ is paired with another multiplier Hopf algebra $A$, we already have that the category of the left modules over $D^{\pi}$ is a braided tensor category, see above. We rephrase the results on this category, but we dispense with the Drinfel'd double $D^{\pi}$ itself. Let $V$ be in ${ }_{B_{p^{-1}}} \pi(\mathcal{Y} \mathcal{D})^{B}$ and $V^{\prime}$ is in ${ }_{B_{q^{-1}}} \pi(\mathcal{Y} \mathcal{D})^{B}$. Then $V \otimes V^{\prime}$ is a $\pi$-Yetter Drinfel'd module over the subalgebra $B_{q^{-1} p^{-1}}$.

For all $p \in G$, there is an invertible function $F_{p}$ on ${ }_{B} \pi(\mathcal{Y D})^{B}$. For $V$ in ${ }_{B_{q^{-1}}} \pi(\mathcal{Y D})^{B}$, we have $F_{p}(V)$ in ${ }_{B} \pi(\mathcal{Y} \mathcal{D})^{B}$. As an algebra, we have that $F_{p}(V)$ equals $V$. As a left $B$-module, $F_{p}(V)$ lies over the subalgebra $B_{p q^{-1} p^{-1}}$. For $b \in B_{p q^{-1} p^{-1}}$ and $v \in F_{p}(V)$, we have $b \cdot F_{p}(v)=F_{p}\left(\pi_{p^{-1}}(b) \cdot v\right)$.

We now find the right $B$-comodule structure on $F_{p}(V)$. If $B$ is paired with a multiplier Hopf algebra $A$, we assume that $W \in M(A \otimes B)$ is the canonical multiplier of this pair. By the uniqueness of the canonical multiplier $W$, we have for all $p \in G$, $\left(\pi_{p}^{\prime} \otimes \pi_{p}\right)(W)=W$. For $v \in V$ and $b \in B$ we have

$$
\begin{aligned}
& \Gamma\left(F_{p}(v)\right)(1 \otimes b)=\sum\left(W^{(1)} \triangleright F_{p}(v)\right) \otimes W^{(2)} b \\
& =\sum F_{p}\left(\pi_{p^{-1}}^{\prime}\left(W^{(1)}\right) \triangleright v\right) \otimes W^{(2)} b=\sum F_{p}\left(W^{(1)} \triangleright v\right) \otimes \pi_{p}\left(W^{(2)}\right) b \\
& =\left(F_{p} \otimes \pi_{p}\right)\left(\sum\left(W^{(1)} \triangleright v\right) \otimes W^{(2)} \pi_{p^{-1}}(b)\right)=\left(F_{p} \otimes \pi_{p}\right)\left(\Gamma(v)\left(1 \otimes \pi_{p^{-1}}(b)\right) .\right.
\end{aligned}
$$

Finally the braiding in the category of left $D^{\pi}$-modules gives the following braiding on ${ }_{B} \pi(\mathcal{Y D})^{B}$. For $V$ in ${ }_{B_{p^{-1}}} \pi(\mathcal{Y} \mathcal{D})^{B}$ and $V^{\prime}$ in ${ }_{B_{q^{-1}}} \pi(\mathcal{Y} \mathcal{D})^{B}$, we have $t_{V, V^{\prime}}: V \otimes V^{\prime} \rightarrow F_{p}\left(V^{\prime}\right) \otimes V$ such that for $v \in V, v^{\prime} \in V^{\prime}$

$$
t_{V, V^{\prime}}\left(v \otimes v^{\prime}\right)=\sum F_{p}\left(W^{(1)} \triangleright v^{\prime}\right) \otimes W^{(2)} \cdot v=\sum F_{p}\left(v^{\prime(1)}\right) \otimes\left(v^{\prime(2)} \cdot v\right) .
$$

In the right hand side of this formula, the tensor $v^{\prime(2)} \cdot v$ should be read as $v^{\prime(2)} b \cdot v$ where $b$ is chosen in $B_{p^{-1}}$ such that $b \cdot v=v$, see [Dr-VD-Z]. The summation 
$\sum v^{\prime(1)} \otimes v^{\prime(2)} b$ stands for the element $\Gamma\left(v^{\prime}\right)(1 \otimes b)$ in $V^{\prime} \otimes B$.

3.2 Remark Suppose that $G$ is given by the trivial group $G=\{e\}$. The $G$-cograded multiplier Hopf algebra $B$ is a usual multiplier Hopf algebra. In the case that $B$ is finite-dimensional (and so $B$ is a Hopf algebra), Theorem 3.1 recovers the categorical interpretation of the usual Drinfel'd double of $B$ which is equivalent with the centre-construction of $B$-mod as given in [K, Section XIII.5].

\subsection{Examples}

\subsubsection{G-cograded multiplier Hopf algebras}

We first give examples of $G$-cograded multiplier Hopf algebras with a crossing.

- The Hopf group-coalgebras and their crossing, as considered in [T], are examples of $G$-cograded multiplier Hopf algebras. This point of view is explained in [A-De-VD, Theorem 1.5]. Let $K(G)$ denote the multiplier Hopf algebra of the complex valued functions with a finite support in $G$. The product is pointwise and the coproduct is dual to the product in the group. We write $K(G)=\bigoplus_{p \in G} \mathbb{C} \delta_{p}$. In this case all the components are equal to the trivial algebra $\mathbb{C}$. The natural crossing on $K(G)$ is related with the adjoint action of $G$ on itself.

- Let $(A, \Delta)$ denote any multiplier Hopf algebra. Let $G$ be a group which acts on the multiplier Hopf algebra $A$ by means of automorphisms $\alpha_{p}$ for all $p \in G$. We assume $\alpha_{e}=\iota, \alpha_{p}\left(\alpha_{q}(a)\right)=\alpha_{p q}(a)$ for all $p, q \in G$ and $a \in A$. Further, the automorphism $\alpha_{p}$ respects the comultiplication of $A$ in the sense that $\Delta\left(\alpha_{p}(a)\right)=\left(\alpha_{p} \otimes \alpha_{p}\right) \Delta(a)$ for all $p \in G$ and $a \in A$. Consider the tensor product algebra $B=K(G) \otimes A$ with the trivial product. In [De1, Example 3.3] is given a non-trivial coproduct on $K(G) \otimes A$ as follows

$$
\Delta\left(\delta_{p} \otimes a\right)\left((1 \otimes 1) \otimes\left(\delta_{q} \otimes a^{\prime}\right)\right)=\sum\left(\delta_{p q^{-1}} \otimes \alpha_{q}\left(a_{(1)}\right)\right) \otimes\left(\delta_{q} \otimes a_{(2)} a^{\prime}\right)
$$

for all $p, q \in G$ and $a, a^{\prime} \in A$.

The multiplier Hopf algebra $B=K(G) \otimes A$ is $G$-cograded. We have $B=$ $\bigoplus_{p \in G} B_{p}$ where $B_{p}=\mathbb{C} \delta_{p} \otimes A$. Let $\left\{f_{p} \mid p \in G\right\}$ denote a family of automorphisms on $A$ which respect the comultiplication of $(A, \Delta)$ and assume furthermore that $f_{p q}=f_{p} \circ f_{q}$ and $f_{p} \circ \alpha_{q}=\alpha_{p q p^{-1}} \circ f_{p}$ for all $p, q \in G$. Then, a crossing of $G$ on 
$B$ is given by the automorphisms $\pi_{p}$ on $B$ where $\pi_{p}\left(\delta_{q} \otimes a\right)=\delta_{p q p^{-1}} \otimes f_{p}(a)$ for all $p, q \in G$ and $a \in A$. Observe that the family $\left\{\alpha_{p} \mid p \in G\right\}$ can always be taken to define a crossing on $B$. In this example all components are equal to the (possible infinite-dimensional) multiplier Hopf algebra $A$. However, the comultiplication on $B$ in not trivially given by the comultiplication on $A$. We notice that $(B, \Delta)$ has integrals if $(A, \Delta)$ has integrals, see [De1, Theorem 1.16.1]. So, in these situations we can consider the pairing $\langle\widehat{B}, B\rangle$ where $\widehat{B}$ denotes the dual multiplier Hopf algebra, in the sense of [VD2]. The pairing $\langle\widehat{B}, B\rangle$ has a canonical multiplier $W$ in $M(\widehat{B} \otimes B)$, see [De-VD2, Proposition 4.12].

\subsection{2 $\pi$-Yetter-Drinfel'd modules}

Let $B$ be a $G$-cograded multiplier Hopf algebra and let $\pi$ denote a crossing of $G$ on $B$. Assume that $\langle A, B\rangle$ is a pair of multiplier Hopf algebras with a canonical multiplier $W$ in $M(A \otimes B)$. The tensor algebra $A \otimes B$ (with trivial product) can be made into a $\pi$-Yetter-Drinfel'd module over $B$ as follows. For all $p \in G$ and $b \in G_{p}$ we set

$$
b \cdot(x \otimes y)=\sum\left(\pi_{p^{-1}}\left(b_{(1)}\right) \triangleright x \triangleleft S^{-1}\left(b_{(3)}\right)\right) \otimes b_{(2)} y
$$

for all $x \in A$ and $y \in B$. Observe that $\downarrow$ and $\triangleleft$ are the regular actions of $B$ on $A$, associated to the pairing $\langle A, B\rangle$.

$$
\Gamma(x \otimes y)((1 \otimes 1) \otimes b)=\sum\left(W^{(1)} x \otimes y\right) \otimes W^{(2)} b
$$

for all $x \in A$ and $y, b \in B$. This $\pi$-Yetter-Drinfel'd module for $B$ corresponds with the left regular module of the Drinfel'd double $D^{\pi}$ on itself.

Let $B$ be a finite-type Hopf group-coalgebra with a crossing $\pi$, in the sense of [T, Section 11]. Then we have $B=\bigoplus_{p \in G} B_{p}$ where for all $p \in G$, the algebra $B_{p}$ is unital and finite-dimensional. This multiplier Hopf algebra $B$ is paired with the (usual) Hopf algebra $A=\bigoplus_{p \in G}\left(B_{p}\right)^{\prime}$ where $\left(B_{p}\right)^{\prime}$ is the linear dual of $B_{p}$. The canonical multiplier $W$ in $M(A \otimes B)$ is given by the formal summation $\sum_{p \in G} f_{p, i} \otimes e_{p, i}$ where $\left\{f_{p, i}\right\} \subset\left(B_{p}\right)^{\prime}$ and $\left\{e_{p, i}\right\} \subset B_{p}$ are dual bases. Consider the tensor algebra $\bigoplus_{p, q \in G}\left(\left(B_{q}\right)^{\prime} \otimes B_{p}\right)$. This algebra is a $\pi$-Yetter-Drinfel'd module for $B$ in the following 
way. For all $p \in G$ and $b \in B_{p}, f \in A$ and $y \in B$ we set

$$
\begin{aligned}
& b \cdot(f \otimes y)=\sum f\left(S^{-1}\left(b_{(3)}\right) \cdot \pi_{p^{-1}}\left(b_{(1)}\right)\right) \otimes b_{(2)} y \\
& \Gamma(f \otimes y)((1 \otimes 1) \otimes b)=\sum_{i}\left(f_{p, i} f \otimes y\right) \otimes e_{p, i} b .
\end{aligned}
$$

\section{References}

[A-De-VD] A.T. Abd El-hafez, L. Delvaux and A. Van Daele, Group-cograded multiplier Hopf (*-)algebras, Algebras and Representation Theory 10(1) (2007), 77-95.

[De1] L. Delvaux, Semi-direct products of multiplier Hopf algebras: smash coproducts, Comm. Algebra 30 (2002), 5979-5997.

[De2] L. Delvaux, On the modules of a Drinfel'd double multiplier Hopf( ${ }_{-}$) algebra, Comm. Algebra 33 (2005), 2771-2787.

[De-VD1] L. Delvaux and A. Van Daele, The Drinfel'd double of multiplier Hopf algebras, J. Algebra 272 (2004), 273-291.

[De-VD2] L. Delvaux and A. Van Daele, The Drinfel'd double versus the Heisenberg double for algebraic quantum groups, J Pure and Appl. Algebra 190 (2004), 59-84.

[De-VD3] L. Delvaux and A. Van Daele, The Drinfel'd double for group-cograded multiplier Hopf algebras, Algebras and Representation Theory, 10(3) (2007), 197-221.

[De-VD-W] L. Delvaux, A. Van Daele and S. Wang, Quasitriangular (G-cograded) multiplier Hopf algebras, J. Algebra 289 (2005), 484-514.

[Dr-VD] B. Drabant and A. Van Daele, Pairing and quantum double of multiplier Hopf algebras, Algebras and Representation Theory 4 (2001), 109-132.

[Dr-VD-Z] B. Drabant, A. Van Daele and Y. Zhang, Actions of multiplier Hopf algebras, Comm. Algebra 27 (1999), 4117-4172. 
[K] C. Kassel, Quantum Groups, Graduate texts in mathematics 155, Springer-Verlag, New York (1995).

[K-T] C. Kassel and V. Turaev, Double construction for monoidial categories, Acta Math. 175 (1995), 1-48.

[M] S. Majid, Doubles of quasitriangular Hopf algebras, Comm. Algebra 19 (1991), 3061-3073.

[T] V.G. Turaev, Homotopy field theory in dimension 3 and crossed groupcategories, Preprint GT/0005291.

[VD1] A. Van Daele, Multiplier Hopf algebras, Trans. Amer. Math. Soc. 342 (1994), 917-932.

[VD2] A. Van Daele, An algebraic framework for group duality, Adv.Math. 140 (1998), 323-366.

[VD-Z1] A. Van Daele and Y. Zhang, A survey on multiplier Hopf algebras. Proceedings of the conference in Brussels on Hopf algebras and Quantum Groups, eds. Caenepeel/Van Oystaeyen (2000), 269-309. Marcel Dekker (New York).

[VD-Z2] A. Van Daele and Y. Zhang, Galois Theory for multiplier Hopf algebras with intergrals, Algebras ans Representation Theory 2 (1999), 83-106.

[Z1] M. Zunino, Double construction for crossed Hopf coalgebras, J. Algebra 278 (2004), 43-75.

[Z2] M. Zunino, Yetter-Drinfel'd modules for crossed structures, J. Pure Appl. Algebra 193 (2004), 313-343. 